\title{
LOS MODELOS DE NEGOCIO Y LA TRANSFORMACION DIGITAL EN LA INDUSTRIA EDITORIAL ESPANOLA
}

\author{
BUSINESS MODELS AND DIGITAL \\ TRANSFORMATION IN THE SPANISH \\ PUBLISHING INDUSTRY
}

Marta Magadán-Díaz

Jesús Rivas-García ${ }^{2}$

\begin{abstract}
RESUMEN
De modo análogo a otras industrias culturales, la industria editorial atraviesa un período de transición y cambio técnico orientado a la implantación del formato digital que cuestiona sus modelos de negocio actuales, obligando a revisar completamente buena parte de los procesos internos, cartera de productos y competencias necesarias para desarrollar su actividad. Esta investigación estudia el impacto de la digitalización sobre la industria editorial española por medio de un estudio de casos realizado sobre tres editoriales seleccionadas a conveniencia de la investigación considerando su peso e importancia relativa. Entre las conclusiones obtenidas se destaca que: a) la producción de obra digital todavía no alcanza el nivel de la obra en papel; b) la fiscalidad del IVA no favorece la oferta de precios competitivos en las obras digitales; c) la incertumbre afecta al modo en que las editoriales españolas encaran la digitalización, adoptando una posición conservadora que no las motiva a liderar el cambio.
\end{abstract}

Palabras clave: Digitalización. Industria editorial. Innovación. Modelo de negocio. Libro electrónico.

\begin{abstract}
Analogously to other cultural industries, the Spanish publishing companies is going through a period of transition and technical change aimed to the implementation of the digital format that questions their current business models, forcing a complete revision of a good part of the internal processes, product portfolio and competences necessary to develop their activity. This research studies the impact of digitalization on the Spanish publishing industry through a case study conducted on three publishers selected at the convenience of the research, considering its weight and relative importance. Among the conclusions obtained, it is highlighted that: a) the digital work production still does not reach the level of the work on paper; b) VAT taxation does not favor the offer of competitive prices in digital works, and c) Uncertainty affects the way in which Spanish publishers approach digitization, adopting a conservative position that does not motivate them to lead the change. Keywords: Digitalization. Publishing industry. Innovation. Business model. Ebook.
\end{abstract}

Artigo recebido em 17/01/2019 e aceito para publicação em 28/07/2019.

1 Doutora em Administração de Empresas pela Universidad de Oviedo, Espanha. Professora da Universidad Internacional de la Rioja, Espanha. CEO na industria editorial, Espanha. E-mail: marta.magadan@unir.net.

2 Doutor em Economía pela Universidad de Oviedo, Espanha. Profesor da Universidad Internacional de la Rioja, Espanha. Director do Mestrado Universitário em Direção de Operações e Qualidade da Universidad Internacional de la Rioja, Espanha. E-mail: jesus. rivas@unir.net. 


\section{INTRODUCCIÓN}

La aparición disruptiva del formato electrónico frente al formato papel en la industria editorial está impactando de tal modo que puede ser considerada como el cambio tecnológico de mayor impacto tras la imprenta de Gutemberg (CARREIRO, 2010), poniendo en cuestión los modelos de negocio tradicionales y forzando a las editoriales a revisar completamente buena parte de los procesos internos, cartera de productos y competencias necesarias para desarrollar su actividad.

Los cambios técnicos radicales operados en la industria son consustanciales a un nuevo espacio de interacción entre agentes económicos caracterizado por su virtualidad y complejidad. Ese nuevo contexto de la economía digital se ha ido construyendo sobre la base de Internet, los dispositivos móviles y las innumerables aplicaciones surgidas al calor de la tecnología (Y00 et al., 2012) que impactan de modo disruptivo, entre otras, sobre la industria editorial, aportando innovaciones cruciales en producto, en procesos y en el diseño de nuevos modelos de negocio (BENGHOZI; LYUBAREVA, 2014; ØIESTAD; BUGGE, 2014). Por tanto, la industria editorial se está transformando y pueden apreciarse nuevos modos de organización de las relaciones entre las diferentes empresas que la conforman.

La teoría y el pensamiento académico en torno a las innovaciones disruptivas ha puesto el foco en la manera en que la tecnología es capaz de redefinir industrias y sectores, de tal modo que los viejos modelos de negocio dominantes acaban por ser reemplazados por otros nuevos, reestructurando, así, toda una industria (CHRISTENSEN, 1997). A menudo, dichos cambios atraviesan diversas etapas o fases antes de que se pueda establecer y consolidar el nuevo diseño dominante (ABERNATHY; UTTERBACK, 1978).

A pesar de que, en general, tanto la tecnología como la conducta del consumidor puede exigir una reorganización industrial, así como una nueva orientación o enfoque hacia el mercado, en la mayoría de las ocasiones, las empresas se ven condicionadas tanto por sus actividades anteriores -su pasado organizacional- como por sus especializaciones actuales. Este condicionamiento las puede abocar a cierto grado de miopía estratégica (LEVITT, 1960) en torno a las tendencias del mercado, a la innovación en procesos y en productos, principalmente (FAGERBERG et al., 2005).

La industria editorial está afrontando una profunda transformación como consecuencia de su transición al mundo digital (MAGADÁN; RIVAS, 2018a), tanto en el tipo de productos que lanza al mercado como en la incorporación de nuevos procesos de producción y distribución (GALUSZKA, 2015), enfrentándose a importantes desafíos como consecuencia de la digitalización (DONOUGHUE, 2010), lo que implicará una restructuración en la forma de publicar (LICHTENBERG, 2011). La industria 
editorial española, como tantas otras, no ha sido ajena a los efectos que sobre ella han provocado las tecnologías. Las organizaciones empresariales que en ella operan han tenido que hacer frente a los nuevos desafíos derivados de innovaciones y cambios tecnológicos relacionados con Internet, el ebook o la impresión bajo demanda, entre otros (MAGADÁN; RIVAS, 2018b).

No es abundante, por el momento, la reflexión académica en torno a la digitalización en el sector editorial, que se ha enfocado generalmente desde la perspectiva de la gestión organizacional (SABATIER; FITZELLE, 2011) o desde la perspectiva industrial (BAJANDOH; ALAMOUDI, 2018).

De modo análogo, la literatura académica en torno a los modelos de negocio estudia el proceso de digitalización desde un enfoque de microgestión (TEECE, 2010). Salvo excepciones (SHATZKIN, 2008; LICHTENBERG, 2011), pocos han sido, hasta el momento, los autores que han establecido un análisis de doble perspectiva, conectando ambos niveles: empresa e industria (ØIESTAD; BUGGE, 2014).

El sector editorial español tiene un importante peso dentro de las denominadas industrias culturales, debido al volumen de negocio generado, al empleo creado y su contribución al PIB, entre otros factores (MAGADÁN; RIVAS, 2018c). España se mantiene como una de las principales potencias editoriales del mundo -la cuarta en producción y facturación-, tras Estados Unidos, Reino Unido y Alemania (MAGADÁN; RIVAS, 2018b).

El objetivo general de esta investigación no es otro que tratar de dar respuesta a la siguiente cuestión: ¿de qué modo los modelos de negocio de las empresas editoriales españolas se ven afectados por la digitalización? Como objetivos específicos este trabajo se propone: a) entender el mecanismo por el que las editoriales adquieren los conocimientos necesarios para poder adaptarse al cambio tecnológico, y b) evaluar la influencia de las políticas culturales o las estructuras de propiedad sobre la transición digital en la industria editorial.

El estudio se conforma del siguiente modo: la sección dos aporta el contexto teórico para el análisis; la tercera sección expone la metodología aplicada; la sección cuatro ofrece el estudio de casos; la sección cinco discute los resultados obtenidos; y la sección seis recoge las conclusiones principales de la investigación.

\section{MARCO TEÓRICO}

Seguidamente, se hace un recorrido por las principales teorías en torno a los modelos de negocio, los procesos de digitalización editorial y la gestión de la innovación, que conforman el marco teórico conceptual de esta investigación. 


\subsection{Los modelos de negocio}

Los modelos de negocio pueden ser entendidos como las hojas de ruta operacionales por las que las organizaciones empresariales dirigen sus productos y servicios a los mercados objetivos con la intención de obtener un beneficio. Sin embargo, dichas hojas de ruta no pueden permanecer aisladas de los procesos de innovación si lo que se pretende es seguir aportando valor a los clientes potenciales (SABATIER et al., 2010).

Es por ello que toda organización empresarial que quiera sobrevivir en su entorno ha de pasar por adaptar su modelo de negocio a todas aquellas nuevas realidades tecnológicas que le permitan mejorar su producto o sus procesos (CHESBROUGH, 2010; TEECE, 2010).

Los modelos de negocio de las organizaciones empresariales conectan, además, a estas con sus respectivos proveedores, distribuidores y clientes (CHESBROUGH, 2010). Esto supone que, más allá de dar forma a la arquitectura de la cadena de valor de la organización, la vincula a otras cadenas de valor, conformado un sistema que trata de dar una respuesta eficaz a las necesidades del mercado (ØIESTAD; BUGGE, 2014).

Los procesos de digitalización adoptados por las empresas editoriales posibilitan nuevos modos de ofrecer contenidos (SHATZKIN, 2008) y promueven, al tiempo, el desarrollo de nuevos modelos de negocio (GORDON et al., 2008; PENG, 2016), adoptando las innovaciones existentes (RAYPORT; SVIOKLA, 1995) para ampliar su cartera de productos (RAYNA; STRIUKOVA, 2016).

Las empresas editoriales, en su búsqueda del éxito competitivo y el desarrollo sostenible, han de adaptarse a los cambios del entorno (CAVALCANTE et al., 2011), por lo que, para aquellas, conocer el enfoque de la innovación en el modelo de negocio implica conectar ambos conceptos en el contexto de la economía actual (ZOTT, AMIT, 2007). Así, en la nueva economía del conocimiento, las organizaciones empresariales serán capaces de detectar nuevas oportunidades de negocio y ventajas competitivas, bien dentro de sus propias estructuras o bien en colaboración con organizaciones digitales especializadas en la innovación del conocimiento (MAGADÁN; RIVAS, 2018a), capaces de renovar y redefinir aquellos sectores maduros dentro de una industria con nuevas ideas, técnicas, productos y servicios, es decir, con nuevas lógicas de generación de valor (MASSA; TUCCI, 2014). 


\subsection{La adquisición de nuevo conocimiento}

El grado en que una organización empresarial es capaz de generar o adoptar nuevo conocimiento condiciona la redefinición de su propio modelo de negocio (AFUAH, 1998).

Es posible mostrar una idea del significado de innovación en producto definiéndola como: a) una nueva tecnología -o combinación de tecnologías- generadora de nuevos productos o servicios para responder a una utilización externa o a una determinada necesidad de mercado y, por tanto, pueden incrementar o expandir el dominio de la empresa (UTTERBACK; ABERNATHY, 1975); b) una nueva tecnología -0 combinación de tecnologías- capaz de mejorar las características, prestaciones y calidad de los productos y servicios ya existentes, o c) una nueva tecnología-o combinación de tecnologías- que anticipa las necesidades del cliente desarrollando productos y servicios que en el momento presente, dicho cliente desconoce necesitar (SCANNELL et al., 2000). No obstante, concentrar los esfuerzos en mejorar exclusivamente los productos y servicios existentes en la cartera de una organización, puede suponer riesgos importantes en el sentido de frenar procesos de innovación más amplios (LEVITT, 1960).

Aunque cualquier tecnología potencialmente disruptiva podría afectar al mecanismo de análisis y evaluación de la cartera de productos de una organización empresarial, es probable que dicha disrupción no se considere necesariamente como un factor de importancia estratégica en el sentido de expandir sus propias bases de conocimiento (SAINIO; PUUMALAINEN, 2007), a pesar de su capacidad de brindar oportunidades para el surgimiento de nuevos productos y procesos y, por lo tanto, pueda resultar el germen para la aparición de nuevos modelos de negocio (SABATIER et al., 2010). En suma, toda empresa que se enfrente a un sistema tecno-económico dinámico debería reevaluar su cartera de productos, así como su modelo de negocio (LOEBBECKE, 2010), y las decisiones adoptadas por aquella evidenciarán su visión sobre la creación de valor y la posición de sus clientes en el nuevo contexto.

Las editoriales han de colaborar con otras empresas -principalmente del sector tecnológicopara dar solución a los problemas y desafíos asociados a los cambios técnicos y socioculturales (PROTOGEROU et. al., 2016), y son estas organizaciones tecnológicas las que pueden adelantarse a los actores tradicionales de la industria editorial en la creación de nuevos modelos de negocio para el contenido digital (LOEBBECKE, 2010). 


\subsection{Digitalización de publicaciones}

Hoy por hoy, Internet y la digitalización están cambiando la economía: a) posibilitando nuevas oportunidades de negocio; b) mejorando la eficiencia de los procesos productivos, y c) desarrollando nuevos mercados (MAGADÁN; RIVAS, 2018a).

El tránsito de lo analógico a lo digital supone desafíos y cambios potencialmente críticos para la industria editorial (CARREIRO, 2010; DONOUGHUE, 2010; LICHTENBERG, 2011). Entre las características esenciales dentro de la publicación digital se pueden señalar tanto la disponibilidad de los contenidos en diferentes formatos como los diversos modos de visualización que dependerán de Ios gustos del consumidor (SHATZKIN, 2008). Al tiempo, las barreras para poder publicar contenidos 0 han desaparecido en unos casos o han disminuido drásticamente en otros (SABATIER; FIZELLE, 2011), lo que está suponiendo el desarrollo de nuevos principios para la producción y distribución de contenido en la economía digital.

Internet facilita que cualquier usuario de la red pueda generar contenidos, favoreciendo su difusión (PENG, 2016). Actualmente, con el desarrollo de las nuevas tecnologías, autores y lectores, pueden contactar directamente sin la necesidad de la intermediación de la empresa editorial, lo que mueve a las editoriales a repensar sus propuestas de valor para aquellos ante la pérdida de control por la eliminación de las barreras de acceso al mercado editorial (MAGADÁN, 2017). Con la adaptación de las empresas editoriales al nuevo contexto digital, es crucial que los editores pongan el foco sobre el cliente (DONOUGHUE, 2010).

Las editoriales, hoy por hoy, no están liderando los procesos de innovación tecnológica que se asumen por otras organizaciones empresariales (BENGHOZI; SALVADOR, 2016). Un ejemplo que evidencia esta afirmación se encuentra en los formatos ePub y PDF para el ebook. Dichos formatos son el resultado de una imposición internacional formulada desde las empresas tecnológicas (ØIESTAD; BUGGE, 2014).

Pero no hay un acuerdo unánime en el hecho de que la digitalización deba modificar inexorablemente los modelos de negocio existentes en la industria editorial. De hecho, es posible que coexistan de modo complementario y no mutuamente excluyente, dentro de una misma empresa editorial, dos modelos de negocio -el tradicional junto al digital-, que la empresa editorial opte por un modelo de negocio híbrido o que, finalmente, se realice una apuesta definitiva por un único modelo de negocio plenamente digital (MARTIN; TIAN, 2016). Tal es así, que el sector editorial no acaba de 
decantarse por una opción específica en su transformación digital (ØIESTAD; BUGGE, 2014), aunque sea consciente de la necesidad de adoptar una decisión al respecto de cara a garantizar su viabilidad futura (LICHTENBERG, 2011), máxime cuando los equilibrios de poder y control sobre el mercado editorial en especial, en la relación autor-editor- se están viendo alterados con los cambios tecnológicos.

Finalmente, el contenido digital deja atrás la distribución física poniendo fin al poder de Ios intermediarios -distribuidoras y librerías- en el mercado. Incluso, las obras en formato papel comercializadas por Internet, se comercializan al margen de las distribuidoras convencionales, bien directamente, bien a través de plataformas -como, por ejemplo, Amazon- que ofrecen al cliente potencial la doble opción de adquirir un determinado contenido en cualquiera de los dos soportes que desee (ØIESTAD; BUGGE, 2014).

\section{METODOLOGÍA}

La metodología utilizada para realizar el estudio empírico es la relativa al estudio de casos. Este enfoque es de naturaleza fundamentalmente cualitativa e interpretativa (CRESSWELL, 2003) y se ajusta al alcance y objetivos de este estudio porque ofrece la posibilidad de explicar o comprender un fenómeno, un proceso o una combinación de estos (CORBETTA, 2003). De hecho, esta metodología resulta ser muy adecuada cuando las preguntas clave son qué (descripción), cómo y por qué (aplicación) en la generación de una teoría (SNOW; THOMAS, 1994).

Las editoriales que componen este estudio fueron seleccionadas según un criterio de conveniencia (accesibilidad y perfil buscado para el conjunto de casos); por lo tanto, dada la dificultad de acceder a profesionales en esta rama, solo se seleccionaron aquellos que podían contribuir efectivamente a la pregunta de investigación, una condición necesaria en los estudios de casos (CRESSWELL, 2003; MILES, HUBERMAN; SALDAÑA, 2013).

Este estudio se apoya en una serie de entrevistas semiestructuradas -combinación de preguntas cerradas y abiertas- a responsables de diferentes departamentos de tres empresas editoriales españolas de diferente tamaño -grande, mediano y pequeño-, seleccionadas conforme a un muestreo por conveniencia (CHETTY, 1996; JONAS, 2018; YIN, 2009) que ha tenido en consideración tanto su peso específico dentro del sector como su trayectoria y prestigio en el desarrollo de proyectos editoriales.

Las diferentes entrevistas se llevaron a cabo tanto de modo presencial como virtual, mediante el uso de Skype, y empleando en cada una de ellas el mismo guion de desarrollo y conducción del 
diálogo, el cual fue elaborado por los investigadores con la intención de poder enfocar adecuadamente las respuestas, pero dejando un margen a la libre expresión de las opiniones de los responsables con el componente abierto del cuestionario empleado para las entrevistas. Se logró, de ese modo, obtener información adicional que, aunque no estaba considerada en el guion original, ha permitido matizar las respuestas y las conclusiones del estudio. El tiempo medio empleado en cada entrevista fue aproximadamente de una hora y fueron realizadas entre enero y octubre de 2017.

Toda la información cualitativa obtenida de las entrevistas realizadas se ha enriquecido y combinado con otras fuentes de información como ferias y encuentros sectoriales, informes sectoriales, diversos medios de comunicación, así como las webs y redes sociales de cada una de las empresas estudiadas. A eso se añadieron otras entrevistas realizadas paralelamente a otros agentes del sector -librerías, distribuidoras y plataformas digitales- conectados con las empresas entrevistadas con objeto de controlar el posible sesgo obtenido de sus respuestas al tiempo que ofrecían puntos de vista alternativos sugerentes e interesantes para los propósitos de este estudio. Esto responde a la necesidad metodológica de una triangulación informativa que evalúe el alineamiento de las respuestas obtenidas de las editoriales a las evidencias documentales existentes y disponibles públicamente.

Conforme el método del estudio de casos, las respuestas recogidas en las entrevistas realizadas fueron analizadas por medio de un proceso de reducción y sistematización comparativa de la información recabada para poder dar respuesta a las cuestiones centrales que forman el objetivo general y los específicos de este estudio y poder dar forma a las conclusiones del mismo (MILES y HUBERMANS, 1994; GERRING y COJOCARU, 2016).

\section{DIGITALIZACIÓN DE EDITORIALES ESPAÑOLAS POR TAMAÑO: ESTUDIO DE CASOS}

En esta sección se expone el estudio de casos relativo al impacto de la digitalización en tres editoriales españolas de diferentes tamaños. Previamente, se muestra una panorámica general de la industria editorial española que pone el acento en sus características únicas.

\subsection{Papeles y competencias en la industria editorial}

Históricamente, la función del editor no ha sido otra que organizar y controlar la producción editorial, desde la llegada del manuscrito hasta su conversión en libro. Es por ello que buena parte 
de las competencias básicas necesarias tenían que ver con: a) la selección y preparación de los manuscritos -diseño, maquetación, corrección orto-tipográfica, principalmente-; b) la organización de los procesos productivos; c) la gestión de derechos; d) el marketing, o e) la pedagogía -adaptación de contenidos a las exigencias normativas en materia educativa-, entre otras. Además, buena parte de estas actividades se desarrollan, en muchos casos, en estrecha colaboración con los autores y/0 sus agentes editoriales. Junto a estos, una amplia gama de profesionales colabora, también, con el editor: equipos de marketing y comercialización, evaluadores de manuscritos, correctores, diseñadores y maquetadores, fundamentalmente.

Todo el proceso editorial comienza con la llegada del manuscrito original a la empresa editorial, al que suceden una serie de etapas y procesos sucesivos que son coordinados por el editor quien supervisa el control presupuestario y temporal de cada uno de los proyectos editoriales (MAGADÁN, 2017).

En la medida en que los riesgos asociados a cada proyecto editorial solo se pueden compensar con volúmenes de venta que resulten adecuados para garantizar una rentabilidad a la organización editorial, se ha ido desarrollando progresivamente una industria global del libro basada en la gestión de derechos para ampliar la comercialización en otras lenguas y países. Y las palancas propulsoras de todo esto son los derechos de propiedad intelectual -derechos de autor cuya gestión se cede normalmente en los contratos editoriales al editor- que se negocian globalmente. Solo en España las traducciones de obras procedentes de otros países suponen el 16,1 \% de la producción total. Por ejemplo, en 2016 se registró un aumento del 7,8 \% en el número de obras traducidas al español respecto del 2015. Por otro lado, la comercialización de derechos editoriales españoles en el extranjero tiene un peso destacado en la estrategia de internacionalización de la industria del libro español (MAGADÁN; RIVAS, 2018a).

\subsection{La digitalización en el ámbito de la empresa}

En esta sección se aporta la información de base de cada una de las tres editoriales españolas seleccionadas para el estudio de casos, poniendo de manifiesto en qué medida cada una de ellas ha sabido sacar provecho de la digitalización en la orientación de sus estrategias a medio y largo plazo. En la tabla 1 se muestra un resumen tanto del perfil de las empresas analizadas como de los principales resultados a las cuestiones planteadas en el estudio de casos sobre el impacto de la digitalización. 
Tabla 1- Impacto de la digitalización

\begin{tabular}{|l|l|l|l|}
\hline Empresa & E1 & E2 & E3 \\
\hline Antigüedad & 58 años & 25 años & 21 años \\
\hline Forma jurídica & $\begin{array}{l}\text { Sociedad } \\
\text { Anónima }\end{array}$ & $\begin{array}{l}\text { Sociedad } \\
\text { Limitada }\end{array}$ & Sociedad Limitada \\
\hline Rango de empleados & $>250$ & entre 5 y 25 & entre 1 y 3 \\
\hline Rango de facturación (en millones de euros) & $>60$ & $0,6-1,5$ & $<0,3$ \\
\hline Títulos publicados & $>1500$ & $>600$ & $>100$ \\
\hline Porcentaje de fondo digitalizado & $100 \%$ & $90 \%$ & $60 \%$ \\
\hline Edición de libro en papel & SI & SI & SI \\
\hline $\begin{array}{l}\text { Distribución directa de libros en papel a través } \\
\text { de su web }\end{array}$ & SI & SI & SI \\
\hline Edición de libro electrónico & SI & SI & SI \\
\hline Formato que más utiliza para el ebook & EPUB & PDF & PDF \\
\hline $\begin{array}{l}\text { Distribución del ebook directamente a través } \\
\text { de su web }\end{array}$ & SI & SI & N0 \\
\hline $\begin{array}{l}\text { Distribución del ebook a través de plataformas } \\
\text { digitales }\end{array}$ & SI & SI & SI \\
\hline $\begin{array}{l}\text { Invertir en la creación de una plataforma digital } \\
\text { propia }\end{array}$ & SI & NO & NO \\
\hline $\begin{array}{l}\text { Asumen que la distribución digital se } \\
\text { concentrará en unas pocas empresas }\end{array}$ & SI & SI & SI \\
\hline $\begin{array}{l}\text { Sector de procedencia de las empresas en las } \\
\text { que se concentrará la distribución digital }\end{array}$ & Tecnológico & Tecnológico & Tecnológico \\
\hline
\end{tabular}

Fuente: elaboración propia.

La editorial E1, constituida en 1959 con forma jurídica de sociedad anónima, tiene una historia de 58 años. Ya desde sus comienzos, orientó su especialización hacia las obras de naturaleza educativa, aunque a partir de las décadas de los setenta y ochenta del pasado siglo va ampliando su visión más allá de libro escolar y desarrolla para ello una estrategia de creación de nuevos sellos editoriales así como la adquisición de otros para extender su campo de acción, lo que la ha llevado a constituirse en todo un grupo editorial con delegaciones en toda España, cotizando en bolsa y afianzando su presencia en Latinoamérica. Actualmente, el Grupo E1, ya integrado en un grupo europeo empresarial desde hace unos quince años, se erige como un importante proveedor de contenidos en ambos formatos, papel y digital. En este último caso, comenzó editando en formato PDF, pero últimamente el ePub es el formato que más emplea, aunque esto depende, lógicamente, de la plataforma que comercializa sus contenidos. Publica algo más de 1500 títulos anualmente. Sus áreas de publicación abarcan: ficción nacional y traducida, literatura infantil y juvenil, materiales formativos escolares y de enseñanza superior, así como revistas de diversa índole, entre otras. 
La editorial E2, constituida en 1992 con forma jurídica de sociedad limitada, tiene una historia de 25 años. Arrancó su actividad con un notable crecimiento, asentado en su sólido y amplio catálogo, que comprende diversas colecciones, tanto en el área de la ficción como en el de la no ficción: desde la novela negra e histórica hasta obras dedicadas al pensamiento político, biografías o estudios sociales e históricos, entre otras. A pesar de que buena parte de su catálogo lo constituyen obras publicadas en castellano, también ha publicado en otras lenguas, como el catalán, el inglés, el francés y el árabe. Publica en torno a unos 600 títulos anuales de media y posee participaciones societarias en cuatro editoriales, una distribuidora y un canal digital de televisión.

La editorial E3, constituida en 1996 con forma jurídica de sociedad limitada, tiene una historia de 21 años. Ya desde sus comienzos se articula en torno a dos sellos editoriales dentro de la misma organización. Uno de los sellos publica obras de muy diversa naturaleza, entre las que se pueden destacar las guías de naturaleza y las de patrimonio artístico, muy valoradas tanto por su riguroso contenido como por la calidad de su material gráfico. Así mismo, se pueden señalar otras colecciones notables en su labor editorial: desde la dedicada a la literatura de viajes hasta la publicación de investigaciones destacadas en los ámbitos de la literatura española e hispanoamericana. Desde hace más de una década, se ha volcado en la poesía, publicando poemarios y antologías de referencia dentro del género en lengua castellana, así como en otros idiomas -siempre con publicaciones bilingües y traducciones cuidadas-. Publica algo más de 100 títulos anualmente, en áreas tan variadas como el ensayo, la biografía, el pensamiento, la narrativa, el cuento y el relato, entre otras. La editorial E3 publica en ambos formatos -papel y electrónico-, pero apuesta decididamente por el libro como objeto cuya materialidad y diseño tienen un valor estético y, por eso, publica ediciones muy cuidadas, prestando especial atención a aspectos tales como: el formato, la tipografía, la encuadernación o las imágenes de la cubierta, que, generalmente, son de autor y elaboradas ex profeso para cada obra. Aunque dispone de todo su fondo editorial digitalizado, solamente comercializa una pequeña parte de aquel por medio de plataformas digitales.

\section{DISCUSIÓN DELOS RESULTADOS}

En esta sección se trata de dar respuesta a las cuestiones planteadas al comienzo del documento y que constituyen tanto el objetivo general como los específicos de esta investigación: ¿de qué modo los modelos de negocio de las empresas editoriales españolas se ven afectados por la digitalización? 
¿A través de qué mecanismos las editoriales adquieren los conocimientos necesarios para poder adaptarse al cambio tecnológico? ¿Cuál es la influencia de las políticas culturales y de las estructuras de propiedad sobre la transición digital de la industria editorial? A continuación, se procederá a sintetizar y discutir los hallazgos obtenidos.

\subsection{Adaptación de tecnologías digitales a modelos de negocio existentes}

Las editoriales analizadas, por una parte, han iniciado los primeros movimientos en la exploración de nuevos productos de base digital, pero por otra, apenas han progresado en el desarrollo de infraestructuras digitales, estándares industriales o sistemas de distribución conjunta, por poner algunos ejemplos. Todavía les queda mucho trayecto por delante antes de que puedan aprovechar plenamente todo el potencial de las tecnologías digitales por medio de nuevos modelos de negocio y estructuras de ventas revisadas.

Aunque en la edición digital desaparece la impresión, hay que tener presentes los nuevos procesos propios de las tecnologías digitales y de la elaboración de ebooks, como la creación de ficheros XMLs o la estructuración de formatos integrando los metadatos, entre otros. Los formatos actuales disponen de etiquetas de metadatos que permiten almacenar la información, como el autor, la editorial, el año o la edición, entre otros. El formato seleccionado ha de ser compatible con el empleado por la plataforma a través de la que se vaya a distribuir el ebook. Las diferencias significativas entre Ios ebooks en formato PDF y aquellos en formato ePub son evidentes a la hora de integrar elementos interactivos que enriquezcan el contenido de la obra digital.

Tanto la divergencia de formatos digitales como las diferentes necesidades y requerimientos establecidos para las obras generalistas frente a las de temática educativa, promueven una percepción de incertidumbre en la industria editorial (MAGADÁN, 2017; MAGADÁN; RIVAS, 2018a). Esta inseguridad provocada por la ausencia de un diseño dominante ha hecho que, hasta el momento, los editores traten de avanzar por terreno trillado experimentando con tecnologías digitales vinculadas a modelos de negocio existentes. En suma, en la medida que se vaya disipando la niebla en torno a las herramientas tecnológicas dominantes, probablemente habrá más interés e incentivo por parte de las editoriales a explorar la posibilidad de desarrollo de nuevos modelos de negocios.

Otra de las preocupaciones de las editoriales está relacionada con la protección de los contenidos y su temor a la piratería, lo que ha llevado a las editoriales a recurrir al DRM. No obstante, 
Ios controles tipo DRM actualmente no están debidamente estandarizados, ni resultan suficientemente accesibles dado su coste. Son muchos los autores y editores que se muestran suspicaces al desarrollo de contenidos digitales ya que perciben que la tecnología, hoy por hoy, no es capaz de proteger debidamente sus contenidos de la piratería. De hecho, la preocupación por parte de los editores es tal que aplican niveles de DRM que limita gravemente el uso y disfrute de los lectores sobre los libros que se adquieren. En definitiva, la negativa de los editores a otorgar a los usuarios similares derechos a los que tendrían con las obras en formato papel ha evidenciado ser una barrera significativa en el desarrollo e implantación de los libros electrónicos.

Aunque los editores todavía siguen aferrados a los modelos de negocio existentes y a los canales de distribución tradicionales, están explorando poco a poco las nuevas oportunidades en la publicación en línea. De hecho, buena parte de las iniciativas y propuestas digitales en las tres editoriales analizadas continúan siendo limitadas y dispersas en términos de sus respectivas organizaciones internas. Los editores estudiados son receptivos, pero no plenamente proactivos, en la digitalización sino, más bien, reactivos: se podría afirmar que están aplicando una combinación fragmentada de varios modelos de negocio; es decir, 'Iadrillos y mortero', 'clics y mortero' y colaborativo y freemium. Sin embargo, el modelo dominante sigue siendo el tradicional de "ladrillos y mortero". Este patrón concuerda con las teorías que subrayan cómo el desarrollo de un elenco o cartera de modelos de negocio paralelos pueden ser una forma de revisar gradualmente los modelos existentes (SABATIER et al., 2010; SMITH et al., 2010).

En la siguiente sección se discute cómo los estudios de casos han evidenciado una escasa adaptación a las expectativas de la demanda privada y de qué modo las instituciones públicas favorecen y preservan el paradigma de publicación analógica.

\subsection{Insuficiente adaptación a las expectativas de la demanda privada}

Los editores investigados han sido conscientes de las distintas expectativas de los usuarios digitales frente a los analógicos. Aunque la obra en formato papel es revisada y reimpresa periódicamente, los usuarios esperan que las obras digitales estén actualizadas con una frecuencia significativamente mayor, que aporten desarrollos y mejoras constantes, así como posibilidades de personalización según los intereses del usuario. Para dar respuesta a esta presión es común, en las industrias de software y servicios, utilizar la creación interactiva de productos: inicialmente, las empresas establecen las 
funcionalidades básicas del producto y, posteriormente, emplean los comentarios y sugerencias de los usuarios para el desarrollo y mejora del producto a lo largo del tiempo, lo que implica actualizaciones constantes y contacto directo con el cliente de cara a poder co-crear un producto cada vez más adaptado a los intereses de los usuarios. En esta línea de trabajo, algunos editores están implementando un nuevo diagrama de flujo en sus respectivos modelos de negocio para sus proyectos digitales de mayor envergadura, aunque siguen siendo la excepción que confirma la regla (MAGADÁN, 2017; MAGADÁN y RIVAS, 2018a).

\subsection{Instituciones que preservan el modelo de publicación analógica}

El modo en que los editores se relacionan con la economía digital digitales y los nuevos modelos de negocio surgidos en tal contexto, también se puede entender en relación con las políticas públicas que rodean a la industria.

El hecho de que se haya establecido en España un tratamiento fiscal diferenciado en el IVA que discrimina los libros por formato, favoreciendo claramente al analógico frente al digital, no solo puede alterar las pautas de consumo en el mercado, sino que dicho tratamiento fiscal puede acabar por convertirse en un obstáculo adicional al proceso de digitalización de las empresas editoriales y su apuesta por las nuevas tecnologías (GESKO, 2013, MAGADÁN; RIVAS, 2019a)

A diferencia de las obras impresas que tributan a un 4\%, los libros electrónicos están sujetos en España a un IVA del 21\%, lo que se traduce en algo tan peculiar como que un mismo contenido se ve perjudicado fiscalmente en un $17 \%$ simplemente porque la empresa editorial decida publicarlo en formato digital. Pero la penalización fiscal se extiende más allá del formato y se contagia a lo largo de la cadena de distribución digital del libro, por lo que la barrera al desarrollo digital no solo impacta sobre las editoriales sino sobre todos los intermediarios digitales que las enlazan con los consumidores potenciales. A todo esto, se añade el hecho de que este tratamiento fiscal discriminatorio atenta contra el principio de neutralidad fiscal, fijado por el Tribunal de Justicia de la Unión Europea por el que, bajo ningún concepto, se puede penalizar fiscalmente la actividad competitiva de los mercados para un mismo producto -contenido- independientemente del medio o forma de distribución (BARRAUD, 2013; GUILLON; THIERRY, 2013; RESPINGUE-PERRIN, 2013; MAGADÁN; RIVAS, 2019a).

Un mercado con el potencial de crecimiento del libro electrónico no puede verse desacelerado por una política fiscal que limite su expansión. Dicho desarrollo es beneficioso porque: a) facilita la 
transición digital en las empresas editoriales; b) extiende la cultura digital en la sociedad incrementando su alfabetización tecnológica y c) favorece la inversión en l+D+i dentro y fuera del propio sector editorial.

Una reducción de la diferencia en el tratamiento fiscal por formatos, en un contexto de precio fijo, no sólo será un factor positivo para las empresas implicadas en la industria del libro, sino que el consumidor final también se verá plenamente favorecido por acceder a contenidos -digitales o en papel- de un modo más económico (MAGADÁN; RIVAS, 2019a).

Por otro lado, no hay una solución clara de las compras públicas de libros electrónicos para las bibliotecas: en función de la plataforma que gane el concurso público, las bibliotecas podrán acceder a unos determinados contenidos digitales, pero a otros no. Tampoco hay un plan de apoyo financiero 0 de ayudas públicas para promover la transición digital y la adaptación tecnológica entre las editoriales españolas (MAGADÁN; RIVAS, 2018a, 2019b).

En cuanto a la inversión pública para promover el hábito de la lectura, hay que indicar que es una condición necesaria pero no suficiente para consolidar una industria que pasa por unos momentos delicados como consecuencia del estancamiento de la facturación del libro en papel y su desubicación ante la disrupción tecnológica proveniente de sectores ajenos al editorial.

De lo indicado anteriormente, se puede concluir que las políticas culturales y las estructuras de propiedad están claramente sesgadas hacia la salvaguarda de los ingresos y la estabilidad económica del régimen de publicación analógica y hacia sus modelos de negocios asociados, limitando considerablemente la digitalización de contenidos. De hecho, las tres editoriales estudiadas reconocen, a través de sus responsables, ver favorecida y promovida la edición en formato papel gracias a la captación de ayudas a la edición física, el concurso en licitaciones para la publicación de obras promovidas por las administraciones territoriales y las compras de obra en papel por parte de bibliotecas públicas, bien directamente o bien indirectamente a través de librerías y distribuidores. En este sentido, los responsables entrevistados consideran que esta política se orienta más a la protección de librerías y distribuidoras de obra en papel, preservando así los canales de distribución tradicional.

\subsection{Implicaciones estratégicas empresariales en la era digital}

Aunque del estudio de casos realizado sobre las tres editoriales seleccionadas se desprende que su comportamiento resulta comprensible y acorde con la teoría revisada, es preciso preguntarse de qué modo los proyectos digitales emprendidos por aquellas afectarán a su futuro desarrollo empresarial. 
A diferencia de lo que acontece en otros ámbitos empresariales estudiados por la literatura académica, las estrategias desarrolladas por la industria editorial en torno a su transformación digital no se han orientado hacia la construcción de barreras de entrada para sus posibles competidores externos (ØIESTAD; BUGGE, 2014). Y probablemente sea esta apertura del mercado lo que permita una mejor y más rápida adopción de las innovaciones disruptivas entre las empresas de la industria editorial española.

El desarrollo del libro electrónico no incentiva a la editorial a reducir su producción de libro en papel, pero sí la puede incentivar a redefinir su modelo de negocio. Las editoriales deberán convivir con dos modelos: el tradicional y el digital. El libro electrónico viene a complementar al libro en papel: no se trata de la sustitución del libro impreso por el ebook. Son productos percibidos como complementarios, no sustitutivos, y las editoriales están buscando fórmulas que potencien ambos formatos, teniendo en cuenta la nueva realidad social y económica.

Internet y las nuevas tecnologías incentivan a las editoriales estudiadas a desarrollar canales directos y cortos para comercializar su fondo editorial. La distribución directa por parte de la editorial ha venido a reforzarse con Internet y las redes sociales (NGUYEN et al., 2015). El desarrollo de las nuevas tecnologías de la información ha supuesto un cambio radical en la forma de relacionarse, de buscar información y, por supuesto, de comprar (SAGHIRI et al., 2017).

Además, con la presencia de Internet las editoriales pueden comercializar sus libros directamente -sin contacto físico con el cliente- y se ofrece más información sobre el libro y el autor. Con la venta de libros a través de su propia web, la editorial reduce los costes implicados en la distribución y venta del fondo editorial y puede ofrecer al cliente la posibilidad de leer algunos capítulos, además de facilitarle toda la información que sea posible como, por ejemplo, precio y disponibilidad.

Aunque podría pensarse que el potencial innovador de la editorial dependería de su capacidad para generar nuevo conocimiento, difundirlo e incorporarlo en nuevos productos, servicios y procesos, el hecho es que la innovación depende, cada vez más, de tecnologías y normas que son externas a esta industria. Hay que señalar que las editoriales perciben que la innovación se relaciona más con inversiones en proyectos y contenidos, que directamente con tecnologías, diseños e innovaciones de procesos, infraestructura y dispositivos. En consecuencia, hay que plantear otro argumento: la editorial podría estar considerando que ha asumido ya tantos riesgos comerciales y estéticos al crear nuevos contenidos que no puede afrontar riesgos técnicos adicionales (DE PROPIS, 2013; BENGHOZI; SALVADOR, 2016) y, por ello, son reacios a invertir directamente en innovaciones, prefiriendo adoptar 
soluciones técnicas contrastadas (PROTOGEROU et al., 2016). Sin embargo, aunque la editorial no lidere procesos de innovación, sí se manifiesta abierta a invertir y adoptar aquellas innovaciones que mejoren la eficiencia de sus procesos internos 0 aumenten el valor añadido final de su producto. En suma, cuanto mayor es el impacto de las innovaciones externas en el sector editorial, mayor sería, a priori, el incentivo de la editorial a invertir en innovación.

El nuevo contexto digital también abre la puerta al desarrollo de nuevos modelos de negocio de los que las empresas editoriales pueden sacar provecho adoptando las innovaciones surgidas al amparo de las tecnologías de la información y la comunicación con objeto de ampliar su cartera de productos.

Sin embargo, por el momento, los editores españoles estudiados -salvo un caso- se están centrando en la publicación de versiones digitales de sus libros en papel en las plataformas de distribución digital, en un intento de minimizar riesgos al apostar por obras contrastadas positivamente en el modelo de negocio tradicional. Esta decisión estratégica conduce, al menos en el corto plazo, a que sean las empresas tecnológicas, como Apple o Google, con suficientes recursos financieros y conocimientos especializados quienes controlen la distribución y venta del libro digital (MAGADÁN y RIVAS, 2018a y 2019b).

\section{CONCLUSIONES}

La transformación digital y las innovaciones juegan un papel clave en la estructura actual del sector editorial. Los editores españoles están adaptando su estrategia editorial a las nuevas necesidades de demanda digital y los nuevos dispositivos tecnológicos emergentes. En estos momentos, los viejos modelos de negocio conviven con los nuevos modelos que surgen. Sin embargo, algunas editoriales no se adaptan al cambio tecnológico o lo hacen a un ritmo más lento de lo que sería deseable.

Las tres editoriales estudiadas, a través de sus responsables, confirman que han ido adquiriendo aquellos conocimientos y competencias digitales necesarios para abordar la transición digital, al tiempo que han tratado de posicionarse adecuadamente para poder dar una respuesta eficaz a los cambios operados en el mercado. Sin embargo, todavía los productos y servicios digitales se perciben como secundarios en comparación a la obra analógica. Tal es así que los sistemas de distribución comercial desarrollados para el libro electrónico emulan la cadena tradicional de la obra en papel. 
Por otra parte, se observa que, en términos relativos, continúa siendo pocas las obras digitales en comparación con las publicadas en formato papel y la explicación de esta situación se encuentra no tanto en la demanda sino en la oferta. De las entrevistas realizadas, se vislumbran las siguientes argumentaciones que estarían detrás de ese escaso desarrollo del fondo digital editorial: a) una escasez 0 ausencia de recursos técnicos y financieros, b) una actitud de aversión al riesgo que hace que las empresas estudiadas se aferren al modelo de negocio tradicional predominante del formato papel, c) una percepción de elevada incertidumbre en torno a ciertos aspectos técnicos (formatos y procesos dominantes), y d) un insuficiente apoyo institucional a través de políticas públicas que promueva la transición digital y la renovación de sus respectivos modelos de negocio.

Hasta ahora, las editoriales analizadas sencillamente se limitan a comercializar las versiones digitales de sus obras en papel más destacadas y exitosas en las plataformas digitales de distribución, con la clara intención de reducir riesgos. Este tipo de decisiones estratégicas está permitiendo el liderazgo de las empresas tecnológicas -con suficientes recursos técnicos y financieros- en la comercialización de los libros electrónicos.

La ausencia de propuestas e iniciativas digitales conjuntas está favoreciendo la entrada de nuevos actores ajenos a la industria del libro -fabricantes de hardware, creadores de software y proveedores de servicios de Internet, entre otros- capaces de ir más allá que los agentes tradicionales de la industria del libro en la generación de nuevos modelos de negocio para los contenidos digitales. Un caso paradigmático es el de Amazon que, dentro del retailing, y con su modelo de cola larga (long tail) va mermando la rentabilidad de los intermediarios clásicos del canal de distribución 0, incluso, los acaba por hacer innecesarios.

A pesar de las limitaciones derivadas de un estudio de casos, sí se observa que la información obtenida de las editoriales analizadas se encuentra alineada con la teoría expuesta. En primer lugar, los editores entrevistados ven el libro electrónico como un bien complementario y no sustitutivo del libro en papel. En segundo lugar, los responsables entrevistados se muestran reticentes a realizar una apuesta firme por el desarrollo de proyecto de I+D porque piensan que ya han asumido suficientes riesgos financieros a apostar por la identificación y publicación de nuevos contenidos. En tercer lugar, las editoriales analizadas prefieren adoptar una posición reactiva asimilando aquellas soluciones técnicas bien probadas y contrastadas técnicamente para introducirlas en sus respectivos modelos de negocio, lo que las lleva a situarse en una evidente posición de dependencia externa en lo tocante a innovación. 
Finalmente, queda plantearse si, en un futuro próximo, la industria editorial española irá un paso más allá en su particular interpretación del cambio tecnológico, que ha decodificado sencillamente en términos de: a) cambio de formato (del papel al electrónico) y b) adaptación parcial de los modelos de negocio tradicional con base en el papel. De momento, a la luz de este estudio de casos, no es posible ver un cambio de actitud que persiga el liderazgo innovador dentro de la propia industria del libro.

\section{REFERENCIAS}

ABERNATHY, W. J.; UTTERBACK, J. M. Patterns of industrial innovation. Technology Review, v. 64, n. 7, p. 254-228, 1978.

AFUAH, A. Innovation management: strategies, implementation and profits. New York: Oxford University Press, 1998.

AZAR, G.; CIABUSCHI, F. Organizational innovation, technological innovation, and export performance: The effects of innovation radicalness and extensiveness. International Business Review, v. 26, n. 2, p. 324-336, 2017.

BAJANDOH, A.; ALAMOUDI, B. Publishing digital books through tablets and smart phones in Saudi Arabia. Journal of Fundamental and Applied Sciences, v. 10, n. 4, p. 317-320, 2018.

BARRAUD, B. Le régime fiscal du livre numérique. Legicom, n. 3, pp. 61-71, 2013.

BENGHOZI, P.; LYUBAREVA, I. When organizations in the cultural industries seek new business models: a case study of the French online press. International Journal of Arts Management, v. 16, n. 3, p. 6-19, 2014.

BENGHOZI, P.; SALVADOR, E. How and where the RyD takes place in creative industries? Digital investment strategies of the book publishing sector. Technology Analysis and Strategic Management, v. 28, n. 5, p. 568-582, 2016.

CARREIR0, E. Electronic books: how digital devices and supplementary new technologies are changing the face of the publishing industry. Publishing Research Quarterly, n. 26, p. 219-235, 2010.

CAVALCANTE, S.; KESTING, P.; UHOI, J. Business model dynamics and innovation. (re)establishing the missing linkages. Management Decision, v. 49, n. 7-8, p. 1327-1342, 2011.

CHETTY, S. The case study method for research in small-and medium-sized firms. International Small Business Journal, v. 15, n. 1, p. 73-86, 1996.

CHRISTENSEN, C. The Innovator's Dilemma. Boston, MA: Harvard Business School Press, 1997.

CORBETTA, P. C. Metodología y técnicas de investigación social. Madrid: McGraw-Hill Interamericana, 2013. 
CRESWELL, J. Educational Research: Planning, Conducting, and Evaluating Quantitative and Qualitative Research (2nd. Ed. ed.). Upper Saddle River: Pearson Education Inc, 2003.

DE PROPRIS, L.. How are creative industries weathering the crisis? Cambridge Journal of Regions, Economy and Society, v. 6, n. 1, p. 23-35, 2013.

DONOUGHUE, P. Beyond the fear of cannibalisation will the book publishing industry survive the digital revolution? Logos, v. 21, n. 3, p. 167-178, 2010.

FAGERBERG, J.; MOWERY, D.; NELSON, R. R. The Oxford handbook of innovation. Oxford: Oxford University Press, 2005.

GALUSZKA, P. Music aggregators and intermediation of the digital music market. International Journal of Communication, n. 9, p. 254-273, 2015.

GERRING, J.; COJOCARU, L. Selecting cases for intensive analysis: a diversity of goals and methods. Sociological Methods Research, v. 45, n. 3, p. 392-423, 2016.

GESKO, M. Factors influencing the microeconomic and fiscal effects of the reduced VAT on books. European Financial Systems 2013. Proceedings of the 10th international scientific conference (pp. 99-106). Brno: Masaryk University. Retrieved from http://is.muni.cz/do/econ/soubory/konference/efs/ Sbornik_2013.pdf, 2013

GORDON, L.; KUNG, D.; DYCK, H. Strategic use of e-commerce in the transformation of the publishing industry. Communications of the International Information Management Association, v. 8, n. 4, p. 65-78, 2008.

GUILLON, 0.; THIERRY, C. Is eBook Pricing Structured to Mirror Paper Book Prices? The cases of France and the USA in 2011. Canadian Journal of Information and Library Science, v. 37, n. 3, p. 207-224, 2013.

HUANG, W.; SWAMINATHAN, J. M. Introduction of a second channel: implications for pricing and profits. European Journal of Operational Research, v. 194, n. 1, p. 258-279, 2009.

JONAS, J. M. Research design. En: Stakeholder Integration in Service Innovation. Wiesbaden: Springer Gabler, p. 17-23, 2018.

KHOUJA, M.; WANG, Y. The impact of digital channel distribution on the experience goods industry. European Journal of Operational Research, v. 207, n. 1, p. 481-491, 2010.

LEVITT, T. Growth and profits through planned marketing innovation. Journal of Marketing, n. 24, p. 1-8, 1960.

LICHTENBERG, J. In from the edge: the progressive evolution of publishing in the age of digital abundance. Publishing Research Quarterly, v. 27, n. 2, p. 101-112, 2011.

LOEBBECKE, C. The emergence of ebooks: just another media industry joining the converging digital world? An explorative study on user preferences and industry structure changes. Washington, DC, Telecommunications Policy Research Conference, 2010. 
MAGADÁN, M. Technological change and innovation in the Spanish publishing industry: organizational effects, Oviedo: Doctoral dissertation, University of Oviedo, 2017.

MAGADÁN, M.; RIVAS, J. Digitization and business models in the Spanish publishing industry. Publishing Research Quarterly, v. 34, n 3, p. 333-346, 2018 a.

MAGADÁN, M.; RIVAS, J. The Impact of Digitization on the Spanish Publishing Industry. The International Journal of the Book, v. 16, n. 2, p. 1-18, $2018 \mathrm{~b}$.

MAGADÁN, M.; RIVAS, J. Variables conductoras de la industria editorial española. El Profesional de la Información, v. 27, n. 6, p. 1335-1345, 2018c.

MAGADÁN, M.; RIVAS, J. "Fiscalidad indirecta del libro en Europa: implicaciones y políticas". EI Profesional de la Información, v. 28, n. 1, p. 1335-1345, 2019a.

MAGADÁN, M.; RIVAS, J. "Adaptación de la industria del libro en España al cambio tecnológico: pasado, presente y futuro de la digitalización”. Información, Cultura y Sociedad, v. 40, p. 31-52, 2019b.

MARTIN, B.; TIAN, X. Books, bytes and business: the promise of digital publishing. New York: Routledge, 2016

MASSA, L.; TUCCI, C. L. Business model innovation. En: The Oxford Handbook of Innovation Management. Oxford: Oxford University Press, p. 420-441, 2014.

MASSA, L.; TUCCI, C. L.; AFUAH, A. A critical assessment of business model research. Academy of Management Annals, v. 11, n. 1, p. 73-104, 2017.

MILES, M.; HUBERMAN, A. Qualitative data analysis. An expanded sourcebook ( $2^{\mathrm{a}} \mathrm{ed}$.). ThousandOaks, CA.: Sage, 1994.

NGUYEN, B.; YU, X.; MELEWAR, TC; CHEN, J. Brand innovation and social media: Knowledge acquisition from social media, market orientation, and the moderating role of social media strategic capability. Industrial Marketing Management, V. 51, P. 11-25, 2015.

ØIESTAD, S.; BUGGE, M. M. Digitisation of publishing: exploration based on existing business models. Technological Forecasting and Social Change, n. 83, p. 54-65, 2014.

PENG, Y. Mobile and digitally-mediated publishing strategies in China: an overview of evolving business models. Publishing Research Quarterly, v. 32, n. 3, p. 247-260, 2016.

PROTOGEROU, A.; KONTOLAIMOU, A.; CALOGHIROU, Y. Innovation in the European creative industries: a firm-level empirical approach. Industry and Innovation, v. 24, n. 6, p. 587-612, 2016.

RAYNA, T.; STRIUKOVA, L. From rapid prototyping to home fabrication: How 3D printing is changing business model innovation. Technological Forecasting and Social Change, n. 102, p. 214-224, 2016.

RAYPORT, J. F.; SVIOKLA, J. J. Exploiting the virtual value chain. Harvard Business Review, v. 73, n. 6, p. 35-51, 1995. 
RESPINGUE-PERRIN, S. Too early, too fast? The regulation of the eBook market in France and its possible effects on EU libraries. Liber Quarterly, n. 23 (2), pp. 81-109,2013.

SABATIER, L. A.; FITZELLE, E. Managing the progressive publishing company during market and technology transitions. Publishing Research Quarterly, n. 27, p. 220-229, 2011.

SABATIER, V.; MANGEMATIN, V.; ROUSSELLE, T. From Recipe to Dinner: Business Model Portfolios in the European Biopharmaceutical Industry. Long Range Planning, n. 43, p. 431-447, 2010.

SAGHIRI, S.; WILDING, R.; MENA, C.; BOURLAKISA, M. Towards a three-dimensional framework for omni-channel. Journal of Business Research, v. 77, p. 53-67, 2017.

SAINIO, L. M.; PUUMALAINEN, K. Evaluating technology disruptiveness in a strategic corporate context: A case study. Technological Forecasting and Social Change, n. 74, p. 1315-1333, 2007.

SCANNELL, T.; VICKERY, S.; DROGE, C. Upstream supply chain management and competitive performance in the automotive supply industry. Journal of Business Logistics, v. 21, n. 1, p. 23-48, 2000.

SHATZKIN, M. Digital publishing in the US: driving the industry to vertical niches? Logos, v. 19, n. 2, p. 56-60, 2008.

SMITH, W. K.; BINNS, A.; TUSHMAN, M. L. Complex Business Models: Managing Strategic Paradoxes Simultaneously. Long Range Planning, n. 43, p. 448-461, 2010.

SNOW, C.; THOMAS, J. Field research methods in strategic management: contributions to theory building and testing. Journal of Management Studies, v. 31, n. 4, p. 457-480, 1994.

TEECE, D. J. Business models, business strategy and innovation. Long Range Planning, n. 43, n 2-3, p. 172-194, 2010.

UTTERBACK, J. M.; ABERNATHY, W. J. A dynamic model of process and product innovation. Omega, n. 35 , p. 639-656, 1975.

YIN, R.. Case study research. Design and methods. $4^{a}$ ed. London: Sage Publications, 2009.

YOO, Y.; BOLAND, R. J.; LYYTINEN, K.; MAJRCZCHA, A. Organizing for Innovation in the digitized world. Organization Science, v. 23, n. 5, p. 1398-1408, 2012.

ZOTT, C.; AMIT, R. Business model design and the performance of entrepreneurial firms. Organization Science, n. 18, p. 181-199, 2007. 